\title{
Stochastic dynamics in molten potassium explored by polarized quasielastic neutron scattering
}

\author{
M. D. Ruiz-Martín, M. Jiménez-Ruiz, and A Stunault \\ Institute Laue Langevin, 6 Rue Jules Horowitz, F-38042-Grenoble Cedex 9, France
}

F. J. Bermejo, R. Fernández-Perea, and C. Cabrillo*

Instituto de Estructura de la Materia, CSIC and Departamento Electricidad y Electrónica-Unidad Asociada CSIC, Facultad de Ciencia y Tecnología, Universidad del País Vasco/EHU, Box 644, E-48080-Bilbao, Spain

(Received 5 June 2007; revised manuscript received 1 August 2007; published 1 November 2007)

The coherent $S_{c}(Q, \omega)$ and single-particle $S_{s}(Q, \omega)$ dynamic structure factors which contribute to the lowenergy spectrum of molten potassium are separated by means of neutron polarization analysis. The linewidth and amplitude of the single-particle spectra follow an apparent sub-Fickian behavior, with a diffusion coefficient below the value found by macroscopic means. Once this is accounted for, the results are found to conform with predictions made from kinetic theory. Also, the available theoretical recipes to account for the coherent quasielastic intensity are seen to reproduce the experimental observations within a region of momentum transfers located about the location where the static structure factor shows its main peak. From the measured data as well as from computer simulation results which are validated by comparison to measurement, a clear separation of time scales involving rapid rattling motions within the liquid cages from diffusional processes involving collective or single-particle rearrangements is established.

DOI: 10.1103/PhysRevB.76.174201 PACS number(s): 61.25.Mv, 66.10. $-\mathrm{x}, 66.30 . \mathrm{Fq}, 65.20 .+\mathrm{w}$

\section{INTRODUCTION}

The nature of low-frequency excitations that give rise to the quasielastic part of the spectrum of density fluctuations $S_{c}(Q, \omega)$ in liquid metals still remains to be understood on a full quantitative basis. Within the hydrodynamic limit $(Q$ $\rightarrow 0)$, the $S_{c}(Q, \omega)$ spectrum can be calculated analytically in terms of linearized hydrodynamics, including first-order corrections. ${ }^{1}$ The basic quantities here are hydrodynamic variables for particle, longitudinal current, and energy densities that are connected with the corresponding conserved quantities. In the simplest case, the spectrum is composed of two finite-frequency peaks corresponding to propagating excitations plus a central, relaxing thermodiffusive mode that shows a linewidth given up to second order in $Q$ by $D_{T} Q^{2}$, where $D_{T}$ stands for the thermal diffusion coefficient. In contrast and also within this limit, the $S_{s}(Q, \omega)$ single-particle counterpart is known to follow an exponential relaxation process, as dictated by Fick's law, with a decay constant given in terms of the $D_{s}$ self-diffusion coefficient, which in frequency space translates into a Lorentzian line shape having a linewidth given by $D_{s} Q^{2}$. Beyond the hydrodynamic realm, the behavior of $S_{s}(Q, \omega)$ is now beginning to be understood on a quantitative basis, ${ }^{2,3}$ mostly as a consequence of the developments of kinetic theories of the mode-coupling family. These have provided us with predictive capabilities able to account for the shape and characteristic parameters (i.e., wave vector dependence of its linewidth and amplitude) of the $S_{s}(Q, \omega)$ spectrum. The picture that emerges from these theoretical approaches, mostly concerning detailed studies carried out for molten sodium, portrays motions within the kinetic regime, that is, for time-length scales comparable with interparticle separations and characteristic times as mediated by collective modes. For temperatures close to melting, coupling of particle diffusion to a longitudinal mode results in a retardation of diffusive motions if compared to the hydrodynamic prescription. ${ }^{3}$ In contrast, diffusive dynamics at higher temperatures is thought to couple to transverse modes.

The state of our knowledge concerning the low-frequency part of $S_{c}(Q, \omega)$ lies, however, on a far more primitive stage. Guidance from kinetic theory ${ }^{4}$ tells us that close to $Q_{p}$, that is, where the static $S(Q)$ structure factor shows its maximum, its linewidth may be approximated by ${ }^{4}$

$$
\Delta \omega_{c}=\frac{2 D_{E} Q^{2}}{S(Q)\left[1-j_{0}(Q \sigma)+2 j_{2}(Q \sigma)\right]},
$$

where $D_{E}$ stands for a hard-sphere diffusion coefficient as given by the Enskog prescription, $\sigma$ characterizes the size of the fluid particles, and $j_{x}$ are spherical Bessel functions. Data given in Ref. 4 for liquified rare gases and molten rubidium show reasonable agreement between linewidths determined by experiment and Eq. (1), as do more recent data on more complex liquids such as $\mathrm{Ga}$, molecular $\mathrm{D}_{2}$, or molten $\mathrm{Ni},{ }^{4,5}$ provided that the value for $D_{E}$ is set to that given by the mass-diffusion coefficient. As a result, one could understand the quasielastic part of $S_{c}(Q, \omega)$ as a self-diffusion-like process of the liquid particles that enables a density fluctuation to relax.

Both the structure and dynamics of molten alkali metals have been analyzed many times using tools developed for hard-sphere systems, since these liquids are believed to constitute one of the closer realizations of such thermodynamic idealization, and therefore, the ionic dynamics of liquid alkali metals is usually considered as the most reliable experimental benchmark against which theoretical or simulational results may be tested. ${ }^{1,6}$

Although low-frequency data for $S_{c}(Q, \omega)$ for the heavier alkali metals such as $\mathrm{Rb}$ and $\mathrm{Cs}$ have been reported, ${ }^{8}$ similar studies concerning the lighter alkali metals $\mathrm{Li}$ and $\mathrm{Na}$ (Ref. 9) mostly rely on computer simulations. This comes as a 
consequence of the difficulty to access this part of the spectrum by means of either inelastic x-ray or neutron scattering. The former technique is at present resolution limited for energy transfers below some $1.5 \mathrm{meV}$, while the neutron scattering cross sections for $\mathrm{Li}$ and $\mathrm{Na}$ are mostly dominated by incoherent-scattering contributions (ratios of coherent to incoherent scattering cross sections of 0.49 and 1.02 , respectively). Notice that in the case of liquid $\mathrm{Na}$ where coherent and incoherent effects have the same neutron cross section, the separation between both single-particle and collective dynamics has been usually achieved by means of a model analysis which is employed to subtract the coherent contribution. ${ }^{3,10}$

Our interest to revisit the dynamics of the lighter alkalis was also motivated by recent measurements on the full spectrum for density oscillations of molten potassium ${ }^{7}$ that has shown the inadequacy of the usual viscoelastic ansatz ${ }^{19}$ to account for the observed line shape of the full $S_{c}(Q, \omega)$ spectra, and in fact, a further level down in the memory function representation of the response function was needed to account for the quasielastic region of $S_{c}(Q, \omega)$. Measurements of the total, coherent plus incoherent contributions to the spectra using an unpolarized neutron beam yielded a set of data where the validity of Eq. (1) seemed to be restricted to the immediate vicinity of $Q_{p}$. In addition, revisiting the dynamics of molten $\mathrm{K}$ is of interest in the light of recent reports, ${ }^{11}$ where an abrupt change in the dynamical properties of liquid alkali metals takes place at the position of $\mathrm{K}$, resulting from a change in the density dependence of the melts if plotted versus the atomic number.

Here, we report on a recent measurement of both $S_{c}(Q, \omega)$ and $S_{s}(Q, \omega)$ performed within the same set of experiments by means of fully polarized neutron quasielastic scattering. Although the ratio of coherent to incoherent neutron scattering for $\mathrm{K}$ is about 6.18 , the coherent intensity, especially at low $Q$ and low frequencies, is too weak to be separated by means of model fits to the total unpolarized spectrum. Such a separation can, however, be achieved by means of the analysis of the changes in spin polarization of a fully polarized neutron beam. Our aim is then to compare the characteristic times involved in both single-particle and collectivediffusion processes as well as the relationship between the wave vector dependence of their corresponding linewidths $\Delta \omega_{c}$ and $\Delta \omega_{s}$. This would serve to attest in much detail the dominant character of the quasielastic peak of $S_{c}(Q, \omega)$ as well as to explore the limits of validity of Eq. (1), which is expected to hold close to $Q_{p}$. In addition to experimental data, computer molecular dynamics (MD) simulations have been carried out with the aim of comparing the obtained results with quantities easily amenable to computation such as the even spectral frequency moments, and it allows us to explore regions in wave vector energy-transfer space difficult to reach by purely experimental means.

\section{EXPERIMENTS}

The experiments were carried out using the IN14 triple axis spectrometer of the Institut Laue-Langevin (Grenoble, France), using the polarization analysis option. The incident beam was polarized with an $s$-bender, and a Heusler analyzer was employed in the detection step. Measurements under high-resolution conditions were carried out with the instrument setup using a fixed incident wave vector $k_{i}=1.97 \AA^{-1}$, which provided an energy resolution of $0.27 \mathrm{meV}$ [full width at half maximum (FWHM)]. The energy-transfer range studied was from -2 to $2 \mathrm{meV}$. A fully polarized beam was employed, having a value of 26.9 for the flipping ratio as derived by direct measurement.

A second set of measurements were carried out, modifying the instrument setup using a higher value for the fixed final wave vector $k_{f}=1.97 \AA^{-1}$, which provided an energy resolution of $0.33 \mathrm{meV}$. The energy-transfer range studied was from -5 to $5 \mathrm{meV}$ in order to study the broad component of the quasielastic line. With this experimental configuration, the measured flipping ratio reached a value of 33 , as evaluated by direct measurement. Particular care was taken to avoid performing measurements using values for $k_{f}$ which involved significant beam depolarization. The final configuration in these second set of experiments thus employed a PG002 reflection from the monochromator (40 min), Soller slits, and a guide field provided by Helmholtz coils.

The instrument measures the spin-flip (sf) and non-spinflip (nsf) double-differential cross sections that are related to the coherent and incoherent cross sections through ${ }^{12}$

$$
\begin{gathered}
\left(\frac{d^{2} \sigma}{d \Omega d E}\right)_{s f}=\frac{2}{3}\left(\frac{d^{2} \sigma}{d \Omega d E}\right)_{i n c}, \\
\left(\frac{d^{2} \sigma}{d \Omega d E}\right)_{n s f}=\frac{1}{3}\left(\frac{d^{2} \sigma}{d \Omega d E}\right)_{i n c}+\left(\frac{d^{2} \sigma}{d \Omega d E}\right)_{c o h},
\end{gathered}
$$

where the subscripts on the right-hand side stand for the incoherent and coherent scattering contributions, respectively. Here, as usual, we make use of the notation adequate for macroscopically isotropic systems, where the momentum and energy transfers are given by

$$
\begin{gathered}
\mathbf{Q}=\mathbf{k}_{\mathbf{i}}-\mathbf{k}, \\
E=\frac{\hbar^{2}}{2 m_{n}}\left(k_{i}^{2}-k^{2}\right),
\end{gathered}
$$

where $m_{n}$ stands for the neutron mass, and $k_{i}$ and $k$ stand for the wave vectors of the incident and scattered radiation, respectively.

The isolation of each one of the two components is then achieved through ${ }^{12}$

$$
\begin{gathered}
\left(\frac{d^{2} \sigma}{d \Omega d E}\right)_{c o h}=\frac{1}{4 K}\left[\frac{3\left(N^{+}-N^{-}\right)}{P_{0}}+\left(N^{+}+N^{-}\right)\right], \\
\left(\frac{d^{2} \sigma}{d \Omega d E}\right)_{i n c}=\frac{3}{4 K}\left[\left(N^{+}+N^{-}\right)-\frac{N^{+}-N^{-}}{P_{0}}\right],
\end{gathered}
$$

where $K$ stands for a common scaling constant and $N$ stands for counts with spin up $(+)$ and spin down $(-)$ states given by 


$$
\frac{2 N^{ \pm}}{K}=\left(1 \pm P_{0}\right)\left(\frac{d^{2} \sigma}{d \Omega d E}\right)_{n s f}+\left(1 \mp P_{0}\right)\left(\frac{d^{2} \sigma}{d \Omega d E}\right)_{s f},
$$

with $P_{0}=P P_{A}(1-D)$ being the effective polarization. Here, $P_{A}$ stands for the efficiency of the Heusler analyzer, $D$ is the beam depolarization, and $P$ is the polarization of the incident beam. Values for $P$ and $P_{A}$ are close to unity, and the beam depolarization is usually small. The three parameters are, however, bound by the value of the flipping ratio $R$ as

$$
P P_{A}(1-D)=\frac{1-R}{R(1-2 f)-1}
$$

where $f$ stands for the spin flipper efficiency which is set to a calibrated value of $f=0.991 \pm 0.003$.

A sample of high-purity metallic potassium was contained within a $\mathrm{Nb}$ cylinder of $10 \mathrm{~mm}$ diameter and $10 \mathrm{~cm}$ height, and molten at $375 \mathrm{~K}$. Constant $Q$ scans were then performed for wave vectors ranging from $0.4 \AA^{-1}$ up to $2.0 \AA^{-1}$. Every data point was measured with the neutron spin flipper located before the sample with flipper-on and flipper-off positions. Finally, the collective and single-particle dynamic structure factors are directly proportional to the respective coherent and incoherent cross sections.

Several measurements carried out for the empty cell showed that within the wave vectors of interest, its contribution, which is mostly coherent $\left(\sigma_{c o h}=6.253\right.$ barns; $\sigma_{i n c}$ $=0.024$ barns), only becomes important at the elastic peak position.

Computer molecular dynamics simulations were performed for an ensemble of 500 atoms at $T=380 \mathrm{~K}$, which is some $43 \mathrm{~K}$ above the melting temperature of the real liquid $\left(T_{m}=336.7 \mathrm{~K}\right)$. We have employed a semiempirical potential $^{13}$ that did reproduce adequately the structure and thermodynamics of both the hot solid and the liquid. An initial fcc crystal configuration was melt at high temperature and equilibrated under NVT conditions with a number density $\rho=0.01269 \AA^{-3}$ that comes close to the experimental estimate. Production runs of 50 ps were carried out in order to compute the properties of interest. Both the calculated $S_{c}(Q, \omega)$ and single-particle $S_{s}(Q, \omega)$ dynamic structure factors were analyzed as a first step, following procedures analogous to those employed to treat experimental data; that is, the latter were analyzed by means of a single central Lorentzian having a linewidth (FWHM) $\Delta \omega_{s}$, while the former involved a central quasielastic Lorentzian of $\Delta \omega_{c}$ plus a contribution to account for the inelastic side peaks. As we refer below, some additional components need to be added to the fit model to reproduce the calculated spectral line shapes.

\section{RESULTS}

\section{A. Experiment}

A set of $I_{c}(Q, \omega)$ and $I_{s}(Q, \omega)$ measured intensities is displayed in Fig. 1 for representative wave vectors below, on top, and past $Q_{p} \approx 1.6 \AA^{-1}$. These quantities are related to the measured cross sections as well as to the two relevant dynamic structure factors through

$$
\left(\frac{d^{2} \Sigma}{d \Omega d E}\right)_{c o h, i n c}=\frac{k_{f}}{k_{i}} \sigma_{c o h, i n c} I_{c, s}(Q, \omega)
$$

$$
I_{c, s}(Q, \omega)=S_{c, s}(Q, \omega) \otimes R(Q, \omega)+\mathrm{bcgr}
$$

where $R(Q, \omega)$ stands for the instrumental resolution function and bcgr is a background term.

All the spectral intensities were approximated in terms of single Lorentzian functions plus deltalike contributions for the residual coherent elastic scattering of the sample container. Data shown in Fig. 2 refer to the incoherent-scattering spectra $S_{s}(Q, \omega)$. The best joint fit for both the linewidth and amplitude yields a subquadratic $Q$ dependence, $\Delta \omega_{s}(Q)$ $=2 D_{e f f} Q^{m}$, yielding a value for the apparent diffusion coefficient $D_{s}^{e f f}=0.26(3) \mathrm{meV}^{m}$ and an exponent $m=1.87(28)$. A quadratic law yields a somewhat smaller value for the diffusion coefficient $D_{s}^{e f f}=0.24(9) \mathrm{meV}^{2}$ with a poorer fit. The value for the diffusion coefficient comes significantly short of the macroscopic value of $0.327 \mathrm{meV} \AA^{2}$ calculated from parametric equations given in Ref. 14. The reasons for such diffusion retardation at microscopic scales is usually attributed to strong coupling with longitudinal collective modes. ${ }^{2,3}$ Put into different words, under the present conditions, mass diffusion only takes place after a relatively large lapse of time, where liquid cages become unable to maintain collective oscillatory motions. The intensity of the peak also follows such a sub-Fickian behavior and is very well accounted for, allowing for a further fitting parameter required as a normalization constant.

The reduction in value of the self-diffusion coefficient with respect to that obtained in the macroscopic limit serves to evaluate the importance of the coupling to collective and slowly varying dynamical modes. In fact, if the diffusion coefficient for a hard-sphere fluid is evaluated from the Enskog prescription $D_{E}$ in terms of the temperature, particle mass, and packing fraction, ${ }^{15}$ the expression ${ }^{3}$

$$
\tilde{M}^{M C}(i \omega=0)=\frac{k_{B} T / M_{k}}{D_{s}^{e f f}}\left[1-\frac{D_{s}^{e f f}}{D_{E}}\right],
$$

where the value for $D_{s}^{\text {eff }}$ is here set to that given by the quadratic fit and $M_{k}$ stands for the atomic mass of $\mathrm{K}$. The quantity $\tilde{M}^{M C}$ yields the mode-coupling contribution to the Laplace transform of the memory function associated with the normalized particle velocity autocorrelation function $\langle v(t) \cdot v(0)\rangle /\left\langle v^{2}\right\rangle$ at zero frequency. The above equation yields a value of $11.2 \mathrm{ps}^{-1}$, which comes to be about twice the value reported for molten $\mathrm{Na}$ at an equivalent temperature. $^{3}$

The departure from Fickian behavior for the linewidth and peak height can partially be accounted for using the firstorder correction given by a mode-coupling estimate valid for low momentum transfers, ${ }^{16}$

$$
\Delta \omega_{s}=D_{s} Q^{2}\left[1-b Q+\mathcal{O}\left(Q^{3 / 2}\right)\right],
$$



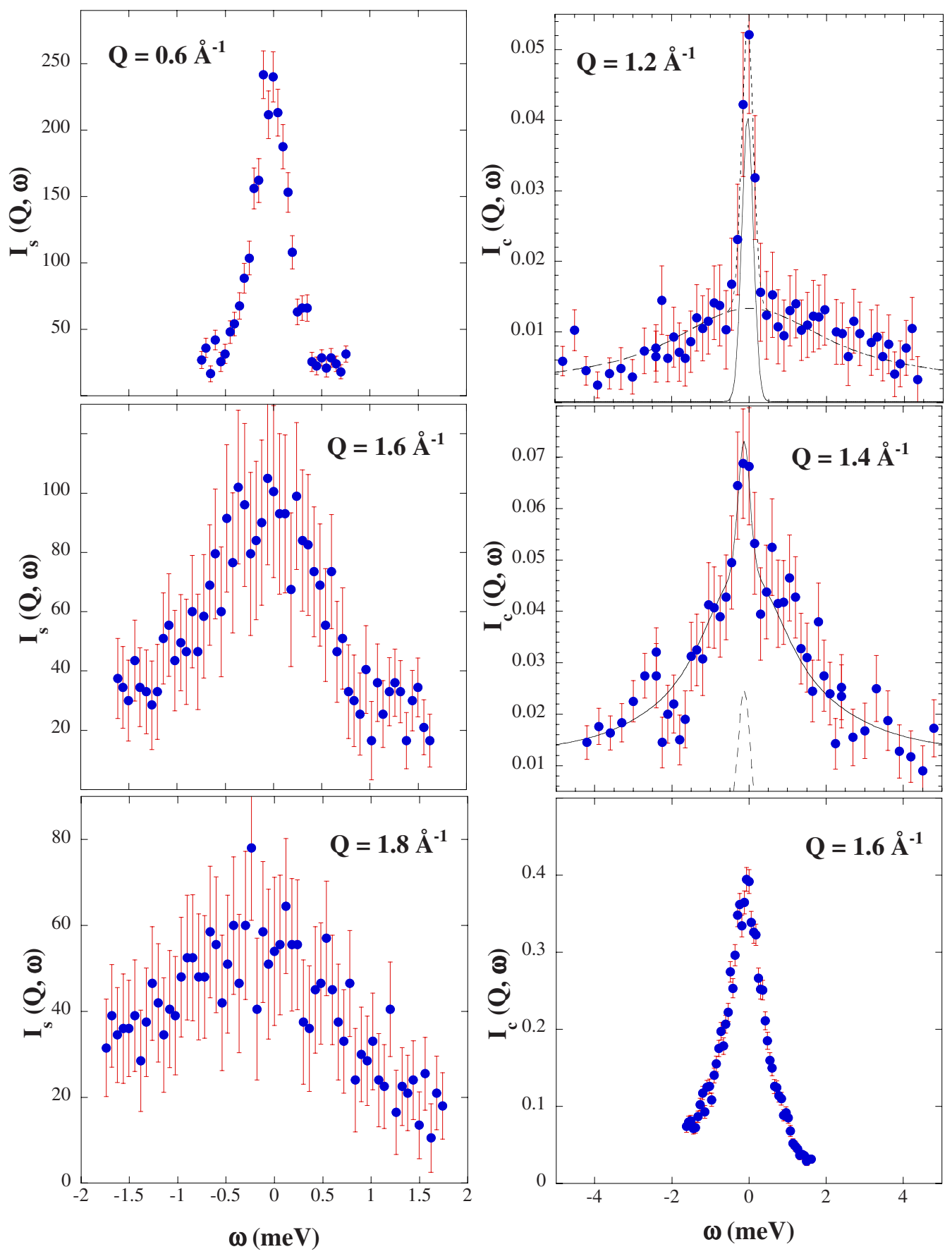

FIG. 1. (Color online) A set of experimental spectra corresponding to single-particle (left side) and coherent (right-hand side) processes. Values for the momentum transfer are given as insets. The intensities for $I_{s}(Q, \omega)$ are given as arbitrary units (neutron counts). Those for $I_{c}(Q, \omega)$ are normalized using the estimate for the static structure factor. Narrow central peaks shown for $I_{c}(Q, \omega)$ arise from residual coherent elastic scattering from the aluminum sample container. The narrow peaks in the two upper frames depict the elastic contribution from the $\mathrm{Nb}$ container.

$$
S_{s}(Q, \omega=0)=\frac{1}{\pi D_{s} Q^{2}}\left[1+a Q^{-1}+\mathcal{O}\left(Q^{3 / 2}\right)\right],
$$

where the two parameters governing the departure from Fickian behavior, namely, $b$ and $a$, can be explicitly calculated from macroscopic properties such as mass density and viscosities as well as from the value of the self-diffusion coefficient. Their values $b=0.0526 \AA$ and $a=0.0969 \AA$ are respectively about $15 \%$ and $17 \%$ above those reported for liquid $\mathrm{Na}$ at an equivalent temperature, where modecoupling effects are known to be already strong. ${ }^{17}$

Data pertaining to the coherent dynamic structure factor are shown in Fig. 3. The amplitude for the fitted Lorentzian closely follows the oscillations shown by the static structure 


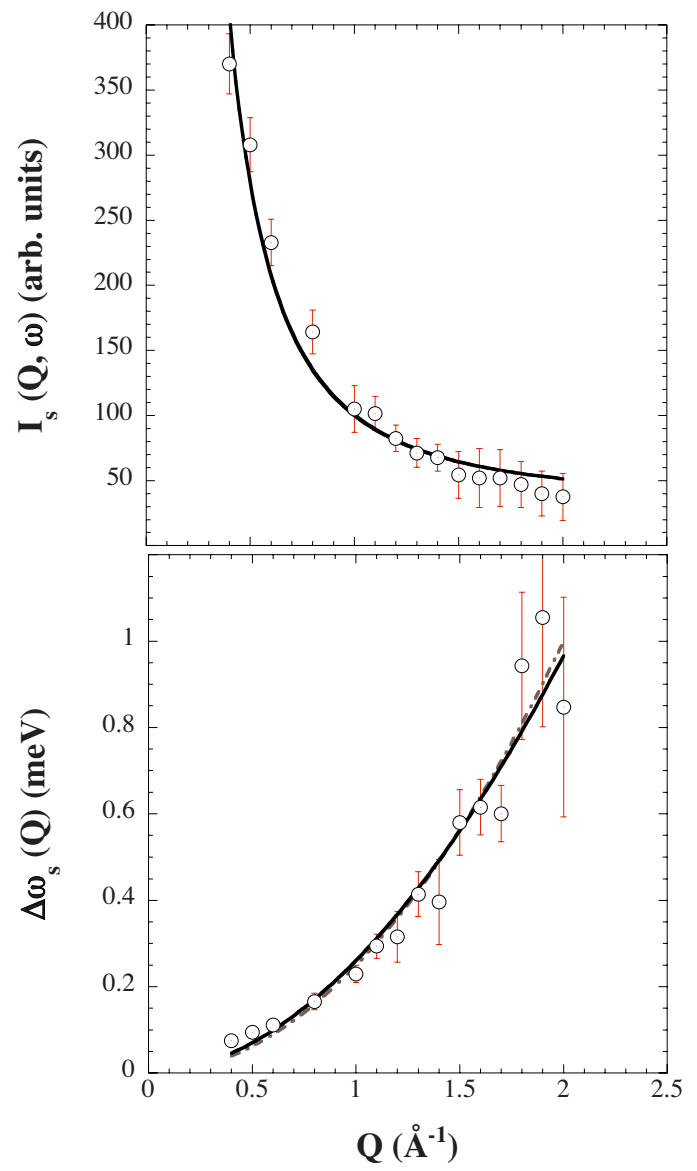

FIG. 2. (Color online) The upper frame displays the measured intensity $I_{S}(Q, \omega=0)$ (symbols) and the solid line displays a fit to $\propto 1 / D_{\text {eff }} Q^{m}$ prediction (see text). The lower frame shows $\Delta \omega_{s}(Q)$ (symbols) together with a fit to $D_{e f f} Q^{m}$. The dash-dot line depicts the best fit achieved using a quadratic, Fickian behavior.

factor, exhibiting a well pronounced maximum at $Q_{p}$. In contrast, its linewidth $\Delta \omega_{c}(Q)$ shows a narrow minimum at $Q_{p}$, and its overall shape consists in an oscillation along the curve giving the $Q$ dependence of the incoherent linewidths. Within the region where Eq. (1) holds, that is, about $Q_{p}$ and somewhat beyond, the linewidth of the coherent component can be reproduced in terms of a single parameter which is set to the value of the experimentally determined self-diffusion coefficient, since a value for the particle diameter of about $4.7 \AA$ was determined from the analysis of a previous inelastic experiment. ${ }^{7}$

\section{B. Computer simulations}

A set of $S_{s}(Q, \omega)$ and $S_{c}(Q, \omega)$ dynamic structure factors were calculated by computer simulation. The analysis of $S_{s}(Q, \omega)$ was first attempted using a single Lorentzian. A second component appears with a small intensity as an inelastic wing, as Fig. 4 clearly shows.

Such a feature arises as a consequence of the markedly sharp peak that appears in the Fourier transform of the particle velocity autocorrelation function $Z(\omega)$ as shown in Ref. 7 , and appears in the calculated spectra as a prominent shoul-

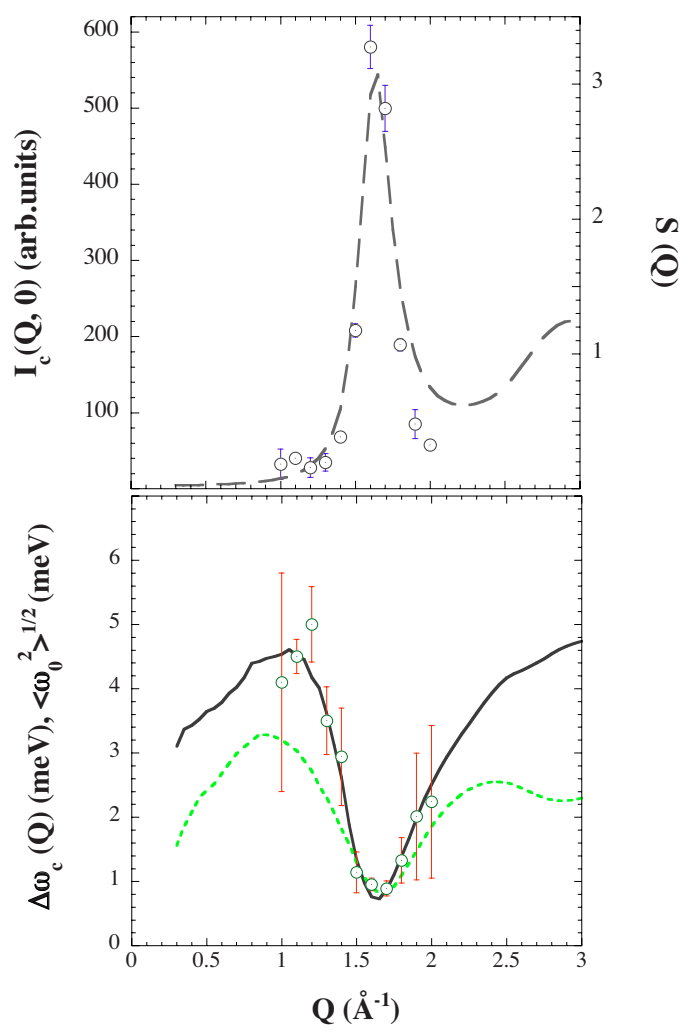

FIG. 3. (Color online) The upper frame displays the intensity $I_{C}(Q, \omega=0)$ (symbols). The results are compared to data pertaining to the static structure factor $S(Q)$ (dashed line). The lower frame shows $\Delta \omega_{c}(Q)$ (symbols) together with a comparison to the prediction made from Eq. (1) using, for the purpose, parameter values of $\sigma=4.7 \AA$ and a value for the diffusion coefficient of $0.24 \mathrm{meV} \AA^{2}$ (solid line). The dashed line depicts the estimated wave vector dependence of the reduced second-frequency moment (see text).

der at about $8 \mathrm{meV}$, which corresponds to the maximum of $Z(\omega)$. Although its contribution to the total spectrum is too weak to be detected by experimental measurements, it does cause a substantial distortion of the line shape if plotted on a semilogarithmic scale. On the other hand, the spectral shape cannot be reproduced in terms of the usual response functions (i.e., Lorentzian, Gaussian, or damped harmonic oscillator, or combinations of those). The difficulty arises from the absence of any robust theoretical expression to account for the shape of $Z(\omega)$ for a liquid metal. In fact, as shown in Fig. 9 of Ref. 7(b), fits using multiparameter expressions such as that derived by Nakahara and Takahashi ${ }^{18}$ can only account for the shape of the frequency distribution on semiquantitative grounds. In consequence, estimates for the quasielastic linewidth were derived by direct measurement of the widths of the spectral distribution rather than from fits to model functions.

From the calculated spectra, the intensity at the peak maxima and linewidth of the quasielastic component of $S_{s}(Q, \omega)$ were measured. Figure 5 displays the wave vector dependence of both the intensity and the linewidth $\Delta \omega_{s}^{M D}(Q)$ of $S_{s}(Q, \omega)$. As regards the latter and in agreement with experiment, data up to relatively large $Q$ may be reproduced using a subquadratic $Q$ dependence, yielding a value for the 


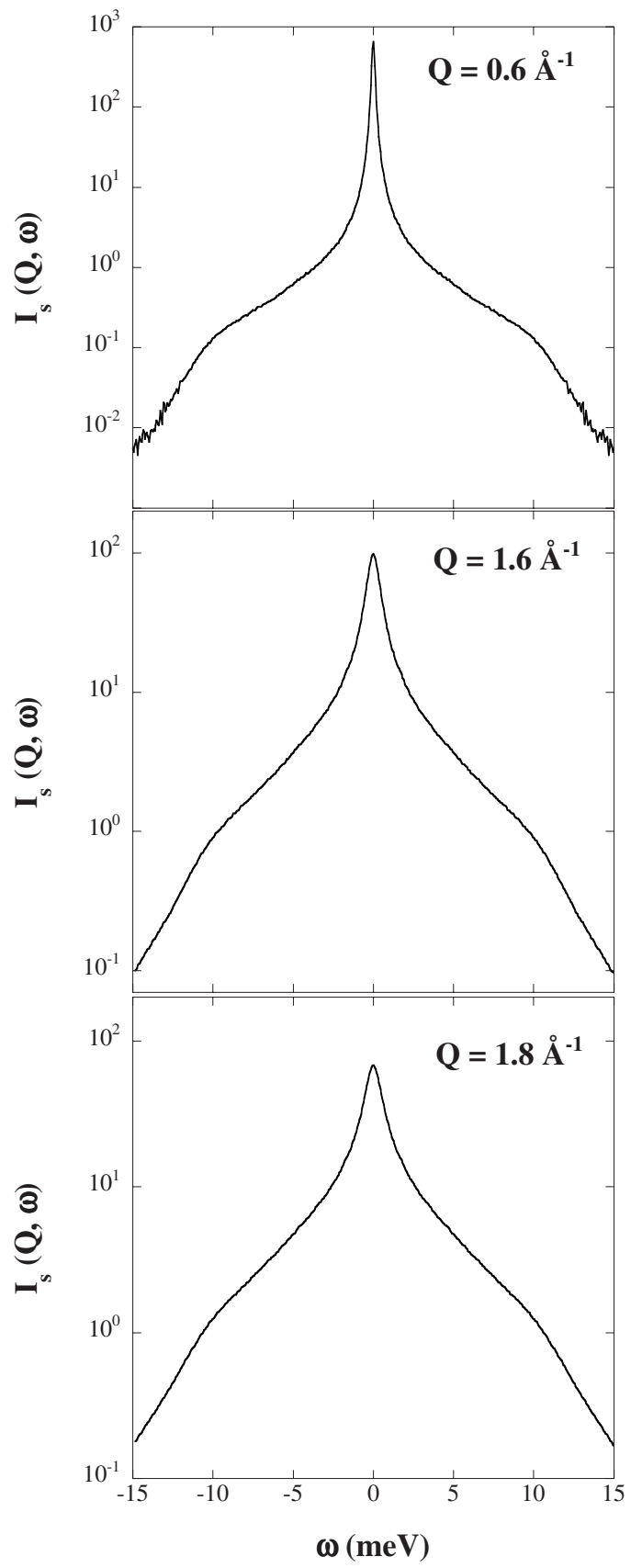

FIG. 4. A set of calculated self-dynamic structure factors comprising the same wave vectors as shown in Fig. 1, plotted on a semilogarithmic scale to allow visualization of the broad inelastic component.

diffusion coefficient of $0.20(2) \AA^{2}$ meV. A strong deviation from such behavior into another having a steeper $Q$ dependence takes place at wave vectors beyond some $2.2 \AA^{-1}$, which is indicative of a change in regime which will, at larger $Q$ 's, approach the free-particle limit in much the same way as observed for molten $\mathrm{Na}$ close to the melting point. ${ }^{3}$

The plot representing the reduced linewidth $\Delta \omega_{s}(Q)^{M D} / D_{s} Q^{2}$ shows that similar diffusion retardation effects to those reported for liquid $\mathrm{Na}$ are operative here. The value estimated for the $a$ coefficient in Eq. (12) comes to be 0.080, which compares to that of 0.0969 derived from ex-

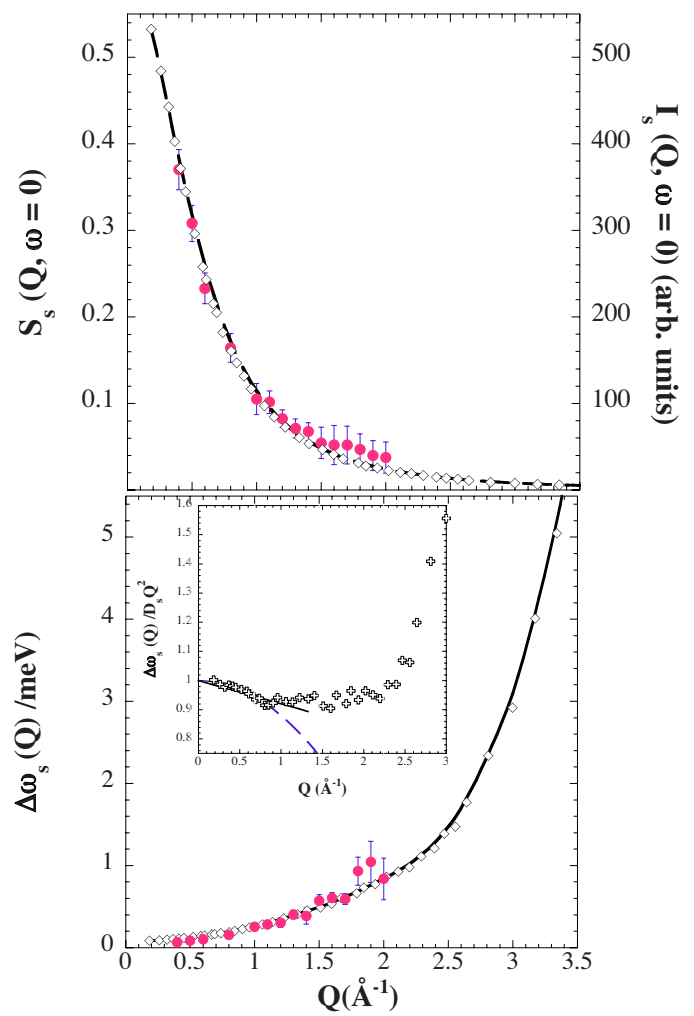

FIG. 5. (Color online) The upper frame displays a comparison of the $Q$ dependence of the calculated incoherent-scattering spectral intensity at zero energy transfer for $S_{s}(Q, \omega)$ (open lozenges) with the experimental intensity (full symbols). The experimental data are scaled to match the simulation. The solid line is drawn as a guide for the eye. The lower frame displays the linewidth as derived from the simulations (open lozenges) together with the experimental estimates (filled symbols). The inset represents the reduced linewidth $\Delta \omega_{s}^{M D}(Q) / D_{s} Q^{2}$ calculated using the value of the derived selfdiffusion coefficient. The straight line represents an approximation at low $Q$ 's as given by Eq. (12). The dashed line represents an approximation to a quadratic deviation as proposed by Wahnström and Sjögren (Ref. 2).

perimental means. Notice, however, that the simulation data suggest that a quadratic deviation following $\Delta \omega_{s} / D_{s} Q^{2}=1$ $-c q^{2}$, with $c=0.12 \AA^{2}$, fits the data significantly better. The issue is, however, of importance since it tells us that coupling to longitudinal collective modes is the main cause for retardation of single-particle motions. ${ }^{3}$

As regards the intensity at zero energy transfers $S_{s}(Q, \omega$ $=0)$ plotted in the upper frame of Fig. 5, it follows a rather similar functional dependence to that shown by experiment, with the obvious exception of the difference in values found for the self-diffusion coefficient.

An example of the simulated spectra for $S_{c}(Q, \omega)$ is displayed in Fig. 6. At the lowest momentum transfer, the spectrum displays the characteristic three-peak shape arising from propagating density oscillations. Our interest here concerns, however, the central, quasielastic region of the spectrum, which we have isolated by approximating the spectral shape to a sum of a central quasielastic peak plus a damped harmonic oscillator function to account for the inelastic fea- 


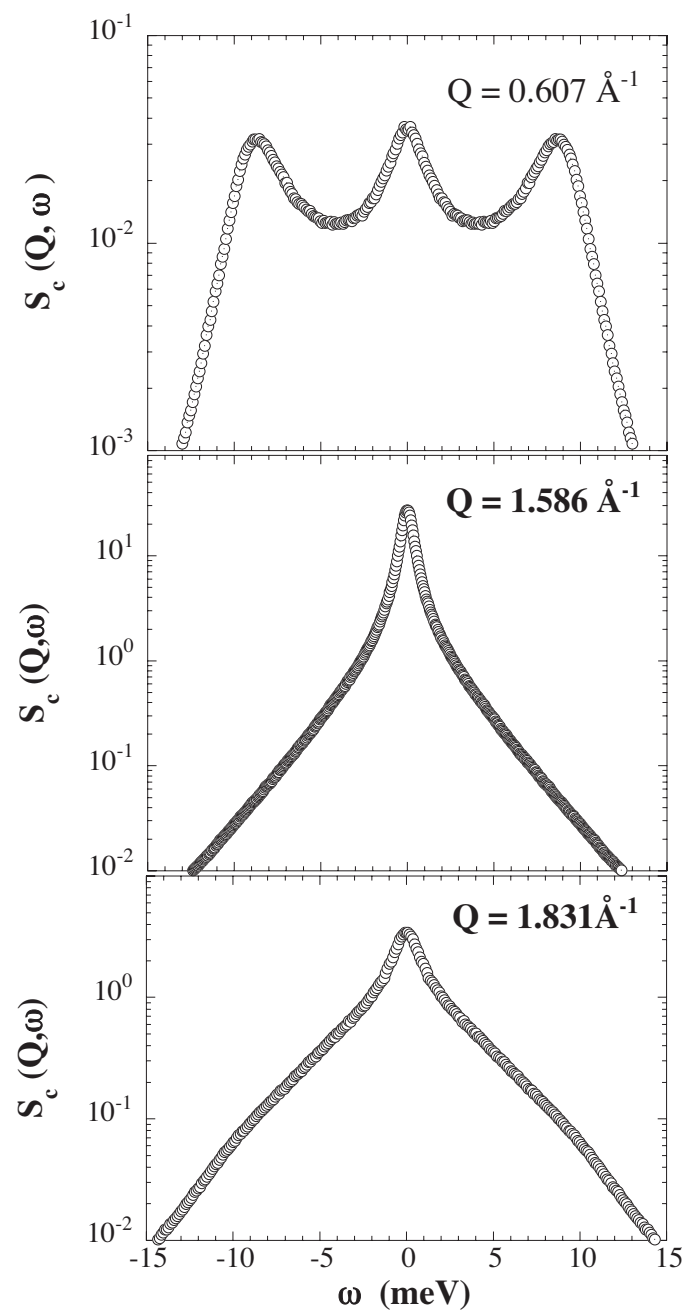

FIG. 6. A set of calculated coherent-dynamic structure factors comprising wave vectors equivalent to those shown in Fig. 4, which represent regions well below the main peak of $S(Q)$, those where $S(Q)$ shows its maximum and wave vectors beyond that.

tures. In this latter respect, the reader is referred to Ref. 7 for a more extended discussion on the collective dynamics taking place at finite frequencies for this liquid.

The $Q$ dependence of the coherent quasielastic linewidth is compared to the present experimental estimate in Fig. 7. Also shown in Fig. 7 are the second reduced frequency moment and the result calculated using the leading order term in Eq. (1) given by ${ }^{19}$

$$
\begin{gathered}
\left\langle\omega_{0}^{2}\right\rangle^{1 / 2}=\frac{Q^{2}}{\beta M_{K} S(Q)}, \\
\Delta \omega_{c}^{*}=\frac{D_{s} Q^{2}}{S(Q)} .
\end{gathered}
$$

Figure 7 also displays a comparison between the experimental data for $\Delta \omega_{c}(Q)$, the value for such a quantity derived from the simulations, and the wave vector dependence and those for the reduced frequency moment. Data shown in Fig.

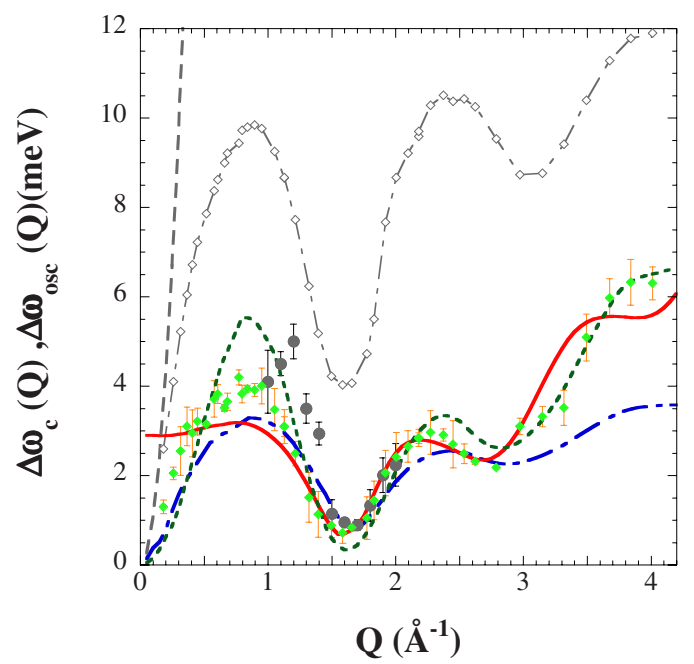

FIG. 7. (Color online) The figure compares the experimental estimates for the coherent quasielastic linewidth (black filled circles) and those derived from simulation (green filled lozenges) to the characteristic frequencies for the finite-frequency excitations (collective density oscillation, open lozenges) as derived from model fits to the simulated spectra. Also shown in the graph are the calculated value for $\left\langle\omega_{0}^{2}\right\rangle^{1 / 2}$ spectral reduced frequency moment (thick dash-dot blue line), the approximation for the simulated data using Eq. (1) (solid red line) which has been calculated using parameters derived from the MD simulation $[\sigma=4.73 \AA$; the leading term in such an approximation (green finely dashed line], and the estimate for the linewidth of the central peak within the hydrodynamic realm calculated using the ionic contribution to the thermal conductivity (dashed line, see text).

7 for $\Delta \omega_{c}(Q)$ show that the simulation significantly underestimates the coherent quasielastic linewidths for wave vectors below $Q_{p}$.

As regards the relative merits of different approximations to estimate the width of the quasielastic spectrum, namely, the reduced second-frequency moment, the leading term to Eq. (1), the three provide close approximations to the data in the vicinity of $Q_{p}$, although $\Delta \omega_{c}^{*}$ significantly underestimates these quantities at $Q_{p}$. As seen from Fig. 7, $\left\langle\omega_{0}^{2}\right\rangle^{1 / 2}$ provides a lower bound for all relevant quantities within most of the explored range of wave vectors. For $Q$ values from $0.6 Q_{p}$ up to a range about $2 Q_{p}$, Eq. (1) constitutes a better approximation to $\Delta \omega_{c}(Q)$ than the other two. This is, however, reversed for $Q$ values below $Q_{p} / 2$, where most experiments dealing with well defined collective oscillations are conducted. Within such a range of wave vectors, the actual values for $\Delta \omega_{c}(Q)$ appear to sit midway between $\Delta \omega_{c}^{*}$ and those given by Eq. (1).

A glance at both Figs. 5 and 7 shows that for wave vectors below $Q_{p}$, mass-diffusion processes involve the longest characteristic time scales, which are somewhat above 10 ps. Such times match those concerning collective particle rearrangements at length scales comparable to $2 \pi / Q_{p}$ as well as at large $Q$ 's where coherent effects vanish. In comparison, motions involving concerted particle rearrangements such as those explored by $\Delta \omega_{c}(Q)$, involve, at $Q_{p} / 2$, about a picosecond. A third time scale involving the propagation of 
acoustic modes involves significantly shorter times as may be gauged by comparison of the upper curve in Fig. 7. The excitation frequencies reach values at the top of the dispersion curve, that is, $Q_{p} / 2$, which amounts to some tenths of a picosecond. In other words, mass diffusion takes place after a rather long period of time has elapsed, which is long enough to allow for the complete decay of the propagating excitation.

A last word concerning the origin of $\Delta \omega_{c}(Q)$ seems now in order. Within the hydrodynamic realm, such a linewidth is given for a simple liquid in terms of the thermal diffusion coefficient $D_{T}=\kappa / \rho M_{k} C_{p}$, where $\kappa$ stands for the macroscopic coefficient of thermal conductivity and $C_{p}$ is the specific heat capacity. From knowledge of the thermodynamic quantities, the derived value for such transport coefficient comes to be, for temperatures close to melting, ${ }^{24} D_{T}$ $=5.36 \AA^{2} \mathrm{eV}$, which is obviously far out of the frequency window explored here. Such a large value arises from the electronic contribution, which swamps the much smaller ionic contribution to such a property. Here, we wish to test a recent suggestion ${ }^{25}$ which attributes to the ionic part of $D_{T}$, at least in part, the origin of the observed coherent quasielastic scattering. Because of the difficulty of accessing such a quantity by experimental means, here we use the recipe derived a long time ago from kinetic theory arguments and states that the ratio of the shear viscosity to the ionic contribution of the thermal conductivity comes as ${ }^{20}$

$$
\frac{\eta_{s}}{\kappa_{\text {ion }}}=\frac{2 M_{K}}{5 k_{B}} .
$$

By using a tabulated value for $\eta_{s}=0.7 \mathrm{cP}$ as well as those concerning the specific heat and mass density, a value for the ionic contribution to $D_{T}$ of $D_{T}^{\text {ion }}=45.05 \AA^{2} \mathrm{meV}$ is derived. Although, as expected, it comes by 2 orders of magnitude below the macroscopic value, its contribution per se to the spectral line shape cannot be large for wave vectors above some $0.1 \AA^{-1}$ as the curve plotted in Fig. 7 showing $D_{T}^{\text {ion }} Q^{2}$ clearly exemplifies.

\section{DISCUSSION AND CONCLUSIONS}

Liquid alkali metals have since long been considered as the closest physical realization of analytically workable models such as those dealing with the uniform distributions of hard spheres. ${ }^{1,21}$ However, in contrast to the transport properties of such idealized systems, the electronic degrees of freedom play here an important role. The data shown here concerning their low-frequency dynamics reveal that the spectrum of such excitations cannot be accounted for in terms of a single time scale attributable to structural relaxation. In fact, even the calculated spectra for single-particle motions display a broad inelastic component arising from a contribution from vibrational states. A similar feature is found in a simulation of molten nickel ${ }^{5}$ as well as in experiments on liquid $\mathrm{Hg} .{ }^{22}$

The main aim of the present study was to quantify in some detail the wave vector dependence of the linewidth of the quasielastic peaks appearing in the collective and single-
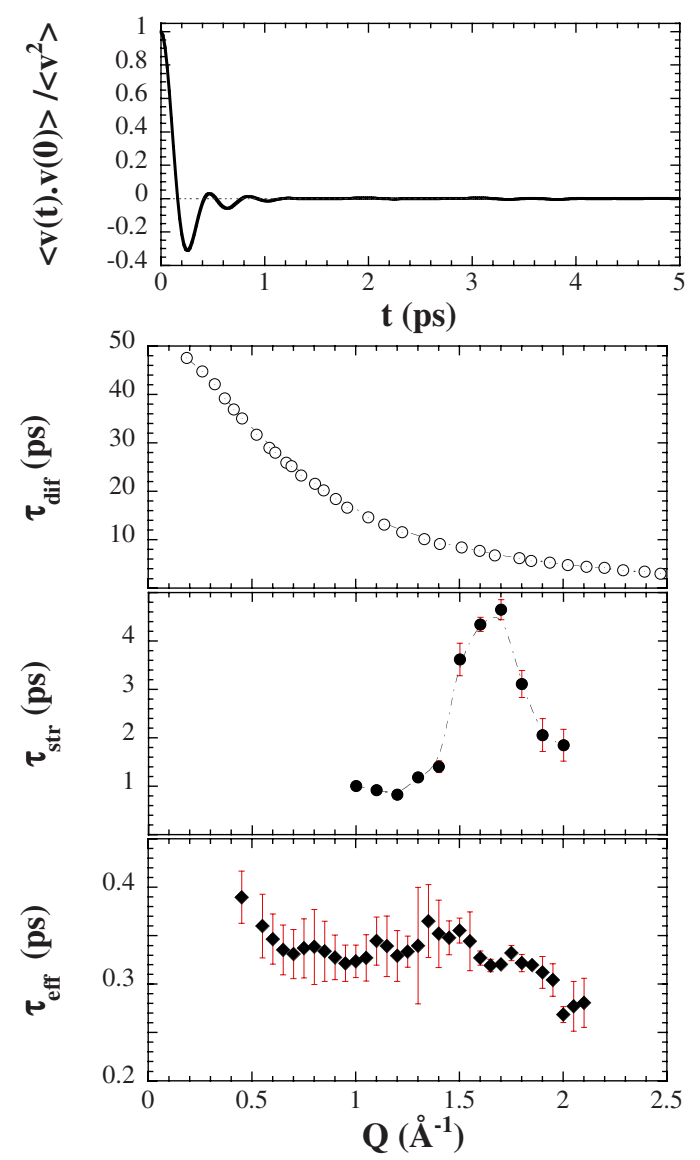

FIG. 8. (Color online) The upper frame displays the normalized particle velocity autocorrelation function as calculated from the simulation results. Frames below depict the characteristic times associated with self-diffusion processes $\tau_{d i f}$ as derived from the present simulation results, the inverse of the coherent quasielastic linewidth $\tau_{\text {str }}$ as calculated from the current set of experiments, and the relaxation time associated with the propagation of longitudinal acoustic excitations $\tau_{\text {eff }}$, which was derived in previous studies (Ref. 7)

particle spectra of a molten alkali metal. With resources at hand, we have been able to separate both contributions to the total spectrum. The picture that emerges from the present study portrays an intimate relationship of the microscopic motions appearing in $S_{c}(Q, \omega)$ to those observable in the single-particle quantity $S_{s}(Q, \omega)$. Furthermore, the achieved separation of both coherent and incoherent scattering components shows that diffusionlike processes either seen through the motions of a tagged particle in $S_{s}(Q, \omega)$ or given in terms of dynamic correlations between diffusing particles by $\Delta \omega_{c}(Q)$ take place within rather different time scales. To quantify such differences in characteristic times, Fig. 8 compares the wave vector dependences of the inverse of the quasielastic incoherent linewidth $\tau_{d i f}(Q)=2 \pi / \Delta \omega_{s}$ as derived from the simulation, the inverse of the coherent quasielastic linewidth $\tau_{s t r}(Q)=2 \pi / \Delta \omega_{c}$ as measured in the present set of experiments, and the relaxation time $\tau_{\text {eff }}$ associated with the propagation of longitudinal acoustic excitations as studied in previous works. ${ }^{7}$ From there, a neat separation in time scales between processes involving particle vibratory oscillations as 
sampled by $\tau_{\text {eff }}$ and mass transport phenomena as those explored through $\tau_{d i f}(Q)$ and $\tau_{s t r}(Q)$ is inferred.

Inspection of graphs showing the characteristic times in Fig. 6 together with the plot showing the normalized particle velocity time correlation function $\mathrm{VACF}=\langle v(t) \cdot v(0)\rangle\left\langle v^{2}\right\rangle$ shows that microscopic vibratory motions take place at times shorter than some $0.5 \mathrm{ps}$, where the VACF exhibits a strong oscillatory structure which includes a strong backscattering (negative) region between 0.15 and $0.45 \mathrm{ps}$, indicative of backflow effects. Such time range compares to the average time between collisions $t_{\text {coll }}=0.17 \mathrm{ps}$ estimated from the thermal velocity $v_{t h}=4.52 \AA \mathrm{ps}^{-1}$ as well as from the mean free path given by $l=\left(\sqrt{2} \pi \sigma^{2} n\right)^{-1} \approx 0.8 \AA$. Relaxation phenomena sensed by quantities such as $\tau_{\text {str }}$ and $\tau_{d i f}$ set in at times where all the oscillatory structures in the VACF have died away. Correlated particle diffusion, that is, the translational motions of particles within the microscopic liquid structures or cages, sets in right after the complete decay of a density oscillation (sound mode). The strong wave vector dependence of $\tau_{s t r}$, which shows a narrow peak where $S(Q)$ has its maximum, suggests assigning these phenomena to "structural relaxation" effects. Notice, however, that long range particle diffusion leading to liquid flow effects takes place at significantly longer times as a comparison of graphs for $\tau_{d i f}$ and $\tau_{\text {str }}$ vividly exemplifies. As a matter of fact, comparison of the two graphs just referred to gives us some clue to understand the success of Eq. (1). Such an expression is deemed to be valid for a range of wave vectors given by 1 $<Q \sigma<\sigma / l$, where $l$ stands for the particle mean free path. For the liquid density here considered, one gets such limits of validity of Eq. (1) to become $0.212 \AA^{-1}<Q<4.7 \AA^{-1}$, which cover a wider range of values than that shown in Figs. 3 and 5 to adequately account for the present data. However, it needs to be noticed that well below $1 \AA^{-1}$, the time scales involved in particle diffusion are 1 order of magnitude larger than those shown by $\tau_{s t r}$ about $Q_{p}$, and therefore, a proper account requires the fulfillment of conservation laws for the local microscopic velocity and energy, which become relevant for density fluctuations having a wavelength well above $\lambda=2 \pi / Q \gg \sigma$, which were not accounted for in the derivation of Eq. (1). ${ }^{4}$
An evaluation of the relevance of heat diffusion processes either arising from the ions or ions plus electrons shows that, contrary to the expectancy that holds within the hydrodynamic realm, the magnitude of the total $D_{T}$ heat diffusion coefficient or even its ionic contribution $D_{T}^{i o n}$ is far too large to significantly contribute to the observed spectrum. Notice, however, that thermal conduction effects are, here, of particular relevance since one expects that a displacement of the electron density motivated by density oscillations will result not only in an electric current but also in a net energy flux. As a result, one should expect a liquid metal to exhibit a coupling between thermal and mechanical degrees of freedom stronger than that found in insulators. Such a scenario was frequently visited through the years, leading to a formal development of mode-coupling theories, ${ }^{23}$ and in fact, explicit calculations for decay rates of heat and viscous flows as well as sound modes revealed the strong interplay between all these quantities as well as the need to account for higher order corrections in the $Q$ dependence of the thermal diffusivity term entering the hydrodynamic equation.

As a concluding remark, the present results show that a minimum of three characteristic relaxation times are needed to account for the microscopic dynamics of a simple liquid as seen from a tagged particle through the $S_{s}(Q, \omega)$ selfscattering and from the $S_{c}(Q, \omega)$ collective structure factors. The result serves to set a yardstick to measure the progress this field has registered since the pioneering studies on liquid $\mathrm{Rb}$ (Ref. 26) and liquid Cs (Ref. 8) where shown to be describable in terms of a single-relaxation time and the line shape could be accounted form in terms of the three-pole formula derived by Lovesey. ${ }^{19}$ Since findings similar to those reported here have already been reported for the lighter alkali metals, ${ }^{3,10}$ one expects that the same two-relaxation time scenario should provide a more complete description of the dynamics of the heavier alkali metals.

Further improvements in instrument development as well as newer calculation approaches are expected to shed some light into some of the pending issues such as the nature of the quasielastic component of $S_{c}(Q, \omega)$ for $Q \ll Q_{p}$, or the role played by the electronic degrees of freedom on microscopic, nonelectronic transport properties. *jbermejo@we.lc.ehu.es

${ }^{1}$ N. H. March, Liquid Metals (Cambridge University Press, Cambridge, England, 1990).

${ }^{2}$ W. Götze and A. Zippelius, Phys. Rev. A 14, 1842 (1976); A. Zippelius and W. Götze, ibid. 17, 414 (1978); G. Wahnström and L. Sjögren, J. Phys. C 15, 410 (1982); U. Balucani and R. Vallauri, Phys. Rev. A 40, 2796 (1989).

${ }^{3}$ C. Morkel and W. C. Pilgrim, J. Non-Cryst. Solids 312-314, 128 (2002); A. Stangl, C. Morkel, U. Balucani, and A. Torcini, ibid. 205-207, 402 (1996); C. Morkel and W. Gläser, Phys. Rev. A 33, 3383 (1986).

${ }^{4}$ E. G. D. Cohen, P. Westerhuijs, and I. M. de Schepper, Phys. Rev. Lett. 59, 2872 (1987). For some recent tests, see C. Mondelli,
M. A. González, F. Albergamo, C. Carbajo, M. J. Torralvo, E. Enciso, F. J. Bermejo, R. Fernández-Perea, C. Cabrillo, V. Leon, and M. L. Saboungi, Phys. Rev. B 73, 094206 (2006); F. J. Bermejo, I. Bustinduy, S. J. Levett, J. W. Taylor, R. FernandezPerea, and C. Cabrillo, ibid. 72, 104103 (2005).

${ }^{5}$ M. D. Ruiz-Martin, M. Jimenez-Ruiz, M. Plazanet, F. J. Bermejo, R. Fernandez-Perea, and C. Cabrillo, Phys. Rev. B 75, 224202 (2007).

${ }^{6}$ T. Scopigno, G. Ruocco, and F. Sette, Rev. Mod. Phys. 77, 881 (2005).

${ }^{7}$ (a) C. Cabrillo, F. J. Bermejo, M. Alvarez, P. Verkerk, A. MairaVidal, S. M. Bennington, and D. Martin, Phys. Rev. Lett. 89, 075508 (2002); (b) J. Phys.: Condens. Matter 16, S309 (2004). 
${ }^{8}$ T. Bodensteiner, C. Morkel, W. Gläser, and B. Dorner, Phys. Rev. A 45, 5709 (1992); F. Demmel, P. Fouquet, W. Häussler, and C. Morkel, Phys. Rev. E 73, 032202 (2006).

${ }^{9}$ A. Torcini, U. Balucani, P. H. K. de Jong, and P. Verkerk, Phys. Rev. E 51, 3126 (1995); U. Balucani and R. Vallauri, Phys. Rev. A 40, 2796 (1989); U. Balucani, A. Torcini, and R. Vallauri, Phys. Rev. B 47, 3011 (1993); T. Scopigno, U. Balucani, G. Ruocco, and F. Sette, Phys. Rev. E 65, 031205 (2002).

${ }^{10}$ W. C. Pilgrim and C. Morkel, J. Phys.: Condens. Matter 18, R585 (2006).

${ }^{11}$ K. N. Lad and A. Pratap, Phys. Rev. E 70, 051201 (2004); Phys. Rev. B 73, 054204 (2006).

${ }^{12}$ W. G. Williams, Polarized Neutrons (Oxford Science, Oxford, 1988), Chap. 5, p. 228.

${ }^{13}$ Potential taken from J. L. Bretonnet and N. Jakse, Phys. Rev. B 50, 2880 (1994).

${ }^{14}$ T. Iida and R. L. Guthrie, The Physical Properties of Liquid Metals (Claredon, Oxford, 1993), p. 201. The quoted value is an average taken over the three data sets here reproduced.

${ }^{15}$ T. E. Faber, Theory of Liquid Metals (Cambdridge University Press, Cambridge, England, 1972), Chap. 3, p. 154.
${ }^{16}$ I. M. de Schepper and M. H. Ernst, Physica A 98, 189 (1979).

${ }^{17}$ W. Montfrooy, I. de Schepper, J. Bosse, W. Gläser, and C. Morkel, Phys. Rev. A 33, 1405 (1986).

${ }^{18}$ Y. Nakahara and H. Takahashi, Proc. Phys. Soc. London 89, 747 (1966).

${ }^{19}$ S. W. Lovesey, Theory of Neutron Scattering in Condensed Matter (Oxford University Press, Oxford, 1984), Vol. I, Chap. 6, p. 210.

${ }^{20}$ H. C. Longuet-Higgins and J. A. Pople, J. Chem. Phys. 25, 884 (1956).

${ }^{21}$ R. Eisenshitz and M. J. Wilford, Proc. Phys. Soc. London 80, 1078 (1962).

${ }^{22}$ Y. S. Badyal, U. Bafile, K. Miyazaki, I. M. de Schepper, and W. Montfrooij, Phys. Rev. E 68, 061208 (2003).

${ }^{23}$ H. E. Stanley, Introduction to Phase Transitions and Critical Phenomena (Oxford University Press, Oxford, 1971), Chap. 15, p. 15.7 .

${ }^{24}$ See Ref. 6, p. 909.

${ }^{25}$ S. Singh and K. Tankeshwar, Phys. Rev. E 70, 013202 (2004).

${ }^{26}$ J. R. D. Copley and S. W. Lovesey, Rep. Prog. Phys. 38, 461 (1975). 